\title{
Fungos Micorrízicos Arbusculares em Dois Fragmentos Florestais de Restinga Periodicamente Inundável em Marambaia, RJ
}

\author{
Rodrigo Camara ${ }^{1}$, Marcos Gervasio Pereira ${ }^{1}$, Cristiane Figueira da Silva ${ }^{1}$, Ranieri \\ Ribeiro Paula ${ }^{2}$, Eliane Maria Ribeiro da Silva ${ }^{3}$ \\ ${ }^{1}$ Departamento de Solos, Universidade Federal Rural do Rio de Janeiro - UFRRJ, Seropédica/RJ, Brasil \\ ${ }^{2}$ Escola Superior de Agricultura Luiz de Queiroz da Universidade de São Paulo - ESALQ, \\ Universidade de São Paulo - USP, Piracicaba/SP, Brasil \\ ${ }^{3}$ Empresa Brasileira de Pesquisa Agropecuária - Embrapa Agrobiologia, Seropédica/RJ, Brasil
}

\begin{abstract}
RESUMO
O presente estudo objetivou avaliar a ocorrência de fungos micorrízicos arbusculares (FMA) em dois fragmentos florestais (FF 1 e FF 2) de Restinga periodicamente inundável, dispostos em um gradiente de saturação hídrica do solo em Marambaia, RJ. Em cada área foram coletadas amostras da camada superficial $(0-5 \mathrm{~cm})$, para avaliação da comunidade de FMA e dos atributos do solo. Entre as seis espécies de FMA encontradas, cinco foram comuns a ambas as áreas e Acaulospora scrobiculata se restringiu a FF 1. Acaulospora e Glomus foram os gêneros mais adaptados às condições ambientais dos ecossistemas. Não houve diferença significativa entre os fragmentos quanto à riqueza de espécies de FMA. Contudo, a abundância de esporos foi maior no FF 1. Parte destes resultados pode ser um reflexo dos menores valores de $\mathrm{P}$ disponível, teor de água e temperatura do solo no FF 1, quando comparado ao FF 2.
\end{abstract}

Palavras-chave: Mata Atlântica, micorriza, solo.

\section{Arbuscular Mycorrhizal Fungi in Two Forest Fragments of Periodically Floodable Restinga at Marambaia, RJ}

\begin{abstract}
The present study aimed to evaluate the arbuscular mycorrhizal fungi (AMF) ocurrence in two forest fragments (FF 1 and FF 2) of periodically floodable Restinga, arranged in a gradient of soil water saturation at Marambaia, RJ. Soil samples were collected at a depth of $0-5 \mathrm{~cm}$ to evaluate the AMF community and soil atributes. Six AMF species were found, five of them occurred in both areas and Acaulospora scrobiculata were restricted to FF 1. Acaulospora and Glomus were the best-adapted genus to the ecosystem conditions. There was no significant difference between the forest fragments in relation to the species richness. However, spores abundancy was higher in FF 1 . Some of these results were influenced by lower values of availability of $\mathrm{P}$, moisture content and temperature in soil at FF 1 , in relation to FF 2.
\end{abstract}

Keywords: Atlantic Rainforest, mycorrhiza, soil. 


\section{INTRODUÇÃO}

Micorriza é uma associação mutualista entre determinados fungos do solo e raízes de plantas imprescindível para o resgate e manutenção da integridade de um ecossistema florestal degradado por atividades humanas (Engel \& Parrotta, 2003). Isto se deve ao fato de que as plantas envolvidas na associação micorrízica recebem benefícios nutricionais (Carneiro et al., 2008; Schiavo et al., 2009), principalmente no caso de solos com baixa fertilidade (Santos et al., 2008). Além disso, a micorrização aumenta a tolerância das plantas a déficits hídricos (Qiangsheng et al., 2006), ao estresse salino (Giri et al., 2007) e aumentam a sua sobrevivência (Dias et al., 2012). Dentre os diferentes tipos de micorrizas, aquelas que envolvem fungos micorrízicos arbusculares (FMA) são as mais comuns na maioria dos ecossistemas terrestres (Brundrett, 2009).

Devido ao seu importante papel ecológico, as micorrizas têm sido empregadas na produção de mudas para a recuperação de áreas degradadas (Souza et al., 2009; Mello et al., 2011; Tavares et al., 2012). Entretanto, existem diferentes graus na compatibilidade fungo microrrízico-planta hospedeira, o que denota uma grande variabilidade na eficiência simbiótica, conforme os simbiontes envolvidos (Pouyu-Rojas et al., 2006). Os benefícios nutricionais conferidos às plantas pela micorrização podem ser maiores com a inoculação de FMA nativos, isto é, aqueles originários de áreas onde as plantas ocorrem naturalmente e estão sujeitas aos fatores de estresse, em comparação com os FMA não-nativos e isolados de áreas onde estes fatores adversos não são encontrados (Wubet et al., 2003; Carneiro et al., 2008).

Desta maneira, o levantamento da comunidade dos FMA nativos em áreas preservadas (RodríguezEcheverría \& Freitas, 2006) possibilitaria a identificação das espécies com potencial para a inoculação de mudas, com vistas à recuperação de áreas degradadas com características semelhantes àquelas. Neste caso, as Restingas devem se constituir foco prioritário em programas de conservação e recuperação da biodiversidade no país, devido ao conhecido grau de degradação em que estas se encontram. O presente estudo objetivou contribuir para o conhecimento da comunidade de FMA em florestas de Restinga periodicamente inundável, a fim de embasar estudos de produção de mudas micorrizadas com estes fungos, para a recuperação de áreas degradadas com características ecológicas semelhantes a estas florestas.

\section{MATERIAL E MÉTODOS}

A Restinga da Marambaia se estende por três municípios: Rio de Janeiro, Itaguaí e Mangaratiba e se localiza sob as coordenadas geográficas $23^{\circ} 03^{\prime} \mathrm{S}$ e $43^{\circ} 36^{\prime} \mathrm{W}$ (Mattos, 2005). Apresenta extensão de $40 \mathrm{Km}$ de comprimento no sentido L-O e área de aproximadamente $49,4 \mathrm{Km}^{2}$ (Roncarati \& Menezes, 2005). O clima na região é tropical chuvoso com inverno seco (Aw) (Köppen, 1948), sendo que a precipitação média anual é de $1.239,7 \mathrm{~mm}$. A temperatura média anual é de $23,7^{\circ} \mathrm{C}$, com a maior temperatura média mensal em fevereiro $\left(26,8^{\circ} \mathrm{C}\right)$, e a menor em agosto $\left(20,9^{\circ} \mathrm{C}\right)$ (Mattos, 2005). O solo foi classificado como Neossolo Quartzarênico Hidromórfico (Menezes \& Araújo, 2005).

A vegetação da Restinga da Marambaia é caracterizada por onze diferentes tipos de formações: quatro herbáceas (herbácea fechada inundável, herbácea fechada inundada, herbácea aberta de praia e herbácea fechada de cordão arenoso), quatro arbustivas (arbustiva aberta não inundável, arbustiva aberta inundável, arbustiva fechada de duna e arbustiva fechada de pós-praia) e três florestais (floresta inundada, floresta inundável e floresta de cordão arenoso) (Menezes \& Araújo, 2005). Para o estudo foram selecionadas duas áreas de floresta de Restinga submetidas à inundação periódica: fragmentos florestais 1 (FF 1) e 2 (FF 2) (Figura 1).

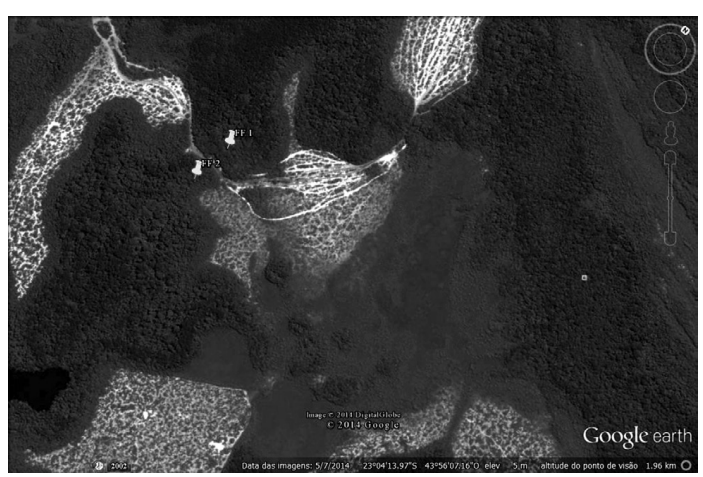

Figura 1. Localização de dois fragmentos florestais (FF 1 e FF 2) em Restinga periodicamente inundável na Marambaia, RJ. Fonte: Google Earth (2014).

Figure 1. Localization of two forest fragments (FF 1 and FF) of periodically floodable Restinga at Marambaia, RJ. Source: Google Earth (2014). 
Tais formações vegetacionais, que se localizam em depressões mal drenadas e apresentam influência do lençol freático, tornam-se encharcadas durante os períodos de maior pluviosidade e comumente se desenvolvem sobre Organossolos, com depósitos turfáceos (Menezes \& Araújo, 2005) que chegam a atingir uma espessura de mais de $20 \mathrm{~cm}$ (Paula et al., 2009). A distância entre FF 1 e FF 2 é de aproximadamente $100 \mathrm{~m}$ e tais áreas estão em um gradiente de saturação hídrica do solo por influência do lençol freático de origem marinha. Nos meses mais secos do ano, são observadas apenas algumas poças esparsas nos pontos mais baixos do relevo em FF 1, enquanto em FF 2 se conserva uma lâmina d'água de cerca de $4 \mathrm{~cm}$ de espessura em toda a sua extensão, no mesmo período, provavelmente devido à sua maior proximidade com o mar (Paula et al., 2009).

Em ambas as áreas a altura média do dossel da comunidade arbórea é de $20 \mathrm{~m}$ de altura, embora ocorram espécies fundamentalmente dominantes e emergentes com alturas superiores a $25 \mathrm{~m}$, que são Tapirira guianensis Aubl. e Calophyllum brasiliense Cambess. Tais fragmentos florestais, que possuem menor diversidade de espécies arbóreas quando comparado com outras formações florestais pertencentes ao bioma Mata Atlântica, apresentam com maior valor de importância praticamente as mesmas espécies: Calophyllum brasiliense (FF 1: 90,2\%; FF 2: 58,5\%), Tapirira guianensis (FF 1: 36,2\%; FF 2: 40,9\%), Tabebuia cassinoides (Lam.) DC. (FF 1: 21,1\%; FF 2: 44,9\%), Myrcia acuminatissima O. Berg (FF 1: 48,2\%; FF 2: 28,2\%), Myrcia multiflora (Lam.) DC. (FF 1: 28,5\%) e Diospyros ebenaster Retz. (FF 2: 38,0\%) (Paula et al., 2009). Os autores do trabalho anteriormente citado salientam que as condições de anaerobiose são responsáveis pelo acentuado acúmulo de serapilheira sobre a superfície do solo e a baixa diversidade de espécies vegetais.

A amostragem foi realizada na estação seca (outubro) de 2006, na camada de $0-5 \mathrm{~cm}$ do solo, próximo da zona radicular de plantas escolhidas ao acaso, em uma área de aproximadamente 1 ha em cada fragmento florestal. Em cada uma destas áreas foram coletadas cinco amostras compostas de solo, cada uma obtida pela reunião de 10 amostras simples de solo, com o auxílio de um anel de PVC com 4,5 cm de diâmetro. No momento da coleta no campo, avaliou-se a temperatura do solo $\left({ }^{\circ} \mathrm{C}\right)$ por meio de geotermômetro digital. Após secagem deste material à sombra por aproximadamente duas semanas, sobre bancada de laboratório, o mesmo foi acondicionado em sacolas de plástico e armazenado em câmara fria $\left(10^{\circ} \mathrm{C}\right)$, até o seu processamento em laboratório (isolamento de esporos de FMA e avaliação do teor de água do solo).

De cada amostra composta retiraram-se $50 \mathrm{~g}$ de solo, para a extração dos esporos de FMA por meio da técnica de peneiramento úmido (Gerdemann \& Nicolson, 1963), seguida de centrifugação em gradiente de densidade (Jenkins, 1964). O material obtido foi vertido para placa de Petri canaletada e observado em microscópio estereoscópico (aumento de 32 x), para avaliação do número total de esporos encontrados (NT) e abundância de esporos $(\mathrm{AB}=$ número médio de esporos em $50 \mathrm{~g}^{-1}$ de solo). Posteriormente, selecionaram-se duas subamostras representativas de toda a variedade de esporos extraídos, segundo as características de tamanho, cor e forma, que foram montadas entre lâmina e lamínula. Destas, uma subamostra foi fixada com álcool polivinil em lactoglicerol (PVLG), e a outra, com a mistura de PVLG + Reagente de Melzer (1:1).

Os esporos foram observados em microscópio ótico com iluminação de campo claro e objetiva de imersão. A identificação das espécies de FMAs foi realizada de acordo com a descrição morfológica disponível na internet na página da International Culture Collection of Arbuscular Mycorrhizal Fungi (http://invam.caf.wwu. edu/). Os caracteres taxonômicos observados foram: número e tipo de camadas das paredes dos esporos e sua reação ao reagente de Melzer; características das paredes internas, quando presentes; morfologia da hifa de sustentação do esporo; e variação da cor e tamanho dos esporos. Desta maneira, estimou-se a riqueza total de FMA (S, número de espécies). A avaliação da comunidade de FMA foi realizada no Laboratório de Micorrizas da Embrapa Agrobiologia (CNPAB).

As avaliações das condições microclimáticas do solo superficial $(0-5 \mathrm{~cm})$ consideraram os valores médios de temperatura e teor de água do solo, para cada fragmento florestal. A temperatura do solo $\left(\mathrm{T},{ }^{\circ} \mathrm{C}\right)$ foi obtida por meio da média dos valores observados em cada ponto de coleta das amostras simples de solo. O teor de água foi avaliado em subamostras das amostras compostas de solo logo após a chegada ao laboratório, por meio da umidade gravimétrica (Ug) $\left(\mathrm{g} \mathrm{g}^{-1}\right)$ seguindo a Fórmula $1: \mathrm{Ug}=[(\mathrm{MU}-\mathrm{MS})] / \mathrm{MS}$, em que: $\mathrm{MU}=$ massa úmida da amostra; $\mathrm{MS}=$ massa seca da amostra, avaliada em balança com duas casas decimais, após permanência da amostra em estufa a $105^{\circ} \mathrm{C}$, por $24 \mathrm{~h}$ (EMBRAPA, 1997). 
Para a avaliação dos atributos químicos do solo, as amostras de solo, após secagem ao ar sobre bancada de laboratório, foram passadas por peneira de 2,00 $\mathrm{mm}$ de diâmetro de malha. Neste material foram analisados os valores de $\mathrm{pH}$ em água e os teores de cálcio + magnésio $\left(\mathrm{Ca}^{+2}+\mathrm{Mg}^{+2}\right)$, alumínio $\left(\mathrm{Al}^{+3}\right)$, potássio $\left(\mathrm{K}^{+}\right)$, hidrogênio + alumínio $\left(\mathrm{H}^{+}+\mathrm{Al}^{+3}\right)$, fósforo assimilável (P) e nitrogênio total $(\mathrm{N})$ (EMBRAPA, 1997), além dos teores de carbono orgânico total (COT) (Yeomans \& Bremner, 1988). As análises laboratoriais foram realizadas no Departamento de Solos do Instituto de Agronomia da Universidade Federal Rural do Rio de Janeiro (UFRRJ), cujos respectivos resultados encontram-se na Tabela 1 .

Os resultados encontrados para nitrogênio total $(\mathrm{N})$, abundância de esporos $(\mathrm{AB})$ e riqueza total de espécies (S) foram avaliados segundo o teste não-paramétrico de Mann-Whitney ou Teste "U” (Zar, 1984). Os resultados obtidos para os demais atributos analisados no solo foram submetidos à análise de variância e, quando não atenderam às suas suposições básicas de normalidade, foram transformados pela expressão $\log +0,5$ ou 1 . $\mathrm{O}$ teste $\mathrm{F}$ da análise de variância foi suficiente para detectar possíveis diferenças entre as áreas. Os dados de $\mathrm{AB}$ foram correlacionados com os dados dos atributos do solo, por meio da correlação de Pearson a $1 \%$ e $5 \%$ de probabilidade. As análises estatísticas foram realizadas pelos programas SAEG 9.0 e Statistica 7.0.

\section{RESULTADOS E DISCUSSÃO}

Nos fragmentos florestais periodicamente inundáveis de Restinga na Marambaia foram encontradas seis espécies de FMA, distribuídas em três gêneros, cada um deles pertencente a uma família: Acaulosporaceae
(Acaulospora foveata Trappe \& Janos; Acaulospora scrobiculata Trappe; Acaulospora sp.), Gigasporaceae (Gigaspora sp.) e Glomeraceae (Glomus macrocarpum Tul. \& C. Tul.; Glomus sp.). Deste total, cinco espécies ocorreram em ambas as áreas estudadas, e apenas Acaulospora scrobiculata se restringiu ao FF 1 . Consequentemente, não foram verificadas diferenças significativas entre FF 1 e FF 2 quanto à riqueza total de espécies de FMA (Tabela 2).

Este resultado foi provavelmente influenciado pela pequena distância que separa os fragmentos florestais estudados (aproximadamente $100 \mathrm{~m}$ ), a qual pode ter favorecido a disseminação cruzada de esporos pelo vento. $\mathrm{O}$ vento é um importante vetor abiótico na dispersão de esporos de FMA em diferentes ecossistemas, como paisagens áridas (Allen et al., 1989), savanas e florestas temperadas, assim como florestas tropicais subtropicais úmidas e estacionais (Egan et al., 2014). A ação deste agente é responsável pela presença de esporos destes fungos até mesmo em dunas costeiras arenosas não-vegetadas (Kulkarni et al., 1997).

No entanto, outros dois fatores podem ter contribuído para a ausência de diferenças entre FF 1 e FF 2 quanto à riqueza de espécies de FMA. Um deles seria as semelhanças entre tais áreas com relação à composição da comunidade arbórea (Paula et al., 2009), uma vez que a abundância de esporos e a riqueza de espécies de FMA varia em função da composição da cobertura vegetal. Esse padrão foi verificado em diferentes ecossistemas arenosos costeiros, como florestas não-inundáveis (Trufem, 1990b; Silva et al., 2012; Souza et al., 2013), áreas de transição floresta-duna (Trufem, 1995) e dunas (Trufem et al., 1989; Trufem, 1990a; Kulkarni et al., 1997; Beena et al., 2001; Cordoba et al., 2001; Jaiswal \& Rodrigues, 2001). O outro fator guarda relação com

Tabela 1. Atributos do solo em dois fragmentos florestais (FF 1 e FF 2) de Restinga periodicamente inundável em Marambaia, RJ. Valores médios de cinco repetições ${ }^{1}$.

Table 1. Soil attributes in two forest fragments (FF 1 and FF 2) of periodically flooded Restinga at Marambaia, RJ. Values are means from five samples ${ }^{1}$.

\begin{tabular}{|c|c|c|c|c|c|c|c|}
\hline \multirow{2}{*}{ Áreas } & pH & $\mathbf{H}^{+}+\mathbf{A l}^{+3}$ & $\mathbf{H}^{+}$ & $\mathbf{A l}^{+3}$ & $\mathrm{Ca}^{+2}$ & $\mathrm{Mg}^{+2}$ & $\mathbf{K}^{+}$ \\
\hline & $\left(\mathrm{H}_{2} \mathrm{O}\right)$ & \multicolumn{6}{|c|}{ 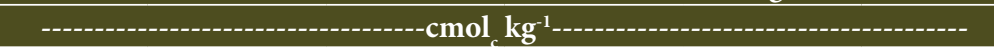 } \\
\hline FF 1 & $4,10 \mathrm{~A}$ & $60,5 \mathrm{~A}$ & $57,5 \mathrm{~A}$ & $2,98 \mathrm{~A}$ & $0,65 \mathrm{~A}$ & $2,26 \mathrm{~A}$ & $0,35 \mathrm{~A}$ \\
\hline FF 2 & $3,89 \mathrm{~A}$ & $63,4 \mathrm{~A}$ & $61,1 \mathrm{~A}$ & $2,35 \mathrm{~A}$ & $0,55 \mathrm{~A}$ & $3,37 \mathrm{~A}$ & $0,42 \mathrm{~A}$ \\
\hline \multirow{2}{*}{ Áreas } & $\mathbf{N a}$ & $\mathbf{P}$ & $\mathbf{N}$ & COT & $\mathbf{C} / \mathbf{N}$ & Ug & $\mathbf{T}$ \\
\hline & $\mathrm{cmol} \mathrm{\textrm {kg } ^ { - 1 }}$ & $\mathrm{mg} \mathrm{kg}^{-1}$ & \multicolumn{3}{|c|}{ 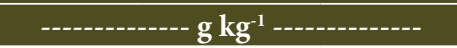 } & $\%$ & ${ }^{\circ} \mathrm{C}$ \\
\hline FF 1 & $0,65 \mathrm{~A}$ & $27,50 \mathrm{~B}$ & $37,17 \mathrm{~A}$ & $346,21 \mathrm{~A}$ & $9,94 \mathrm{~A}$ & $3,50 \mathrm{~B}$ & $21,54 \mathrm{~B}$ \\
\hline FF 2 & $0,52 \mathrm{~A}$ & $51,08 \mathrm{~A}$ & $40,32 \mathrm{~A}$ & $333,99 \mathrm{~A}$ & $8,83 \mathrm{~B}$ & $5,36 \mathrm{~A}$ & $22,68 \mathrm{~A}$ \\
\hline
\end{tabular}

${ }^{1}$ Médias seguidas de letras distintas na coluna são significativamente diferentes (teste $\mathrm{U}$ de Mann-Whitney, $\mathrm{p}<0,05$ ). 
Tabela 2. Estrutura da comunidade de fungos micorrízicos arbusculares no solo em dois fragmentos florestais (FF 1 e FF 2) em Restinga periodicamente inundável em Marambaia, RJ. Valores médios de cinco repetições ${ }^{1}$.

Table 2. Structure of arbuscular mycorrhizal fungi community in two forest fragments (FF 1 and FF 2) of periodically flooded Restinga at Marambaia, RJ. Values are means from five samples ${ }^{1}$.

\begin{tabular}{|c|c|c|c|c|}
\hline NT & AB & $\mathbf{S}$ & Família & Espécie \\
\hline \multicolumn{5}{|c|}{ FF 1} \\
\hline $1283 \mathrm{~A}$ & $\begin{array}{l}256,60 \mathrm{~A} \\
( \pm 53,02)\end{array}$ & $6 \mathrm{~A}$ & $\begin{array}{c}\text { Acaulosporaceae } \\
\text { Gigasporaceae } \\
\text { Glomeraceae }\end{array}$ & $\begin{array}{l}\text { Acaulospora foveata Trappe \& Janos } \\
\text { Acaulospora scrobiculata Trappe } \\
\text { Acaulospora sp. } \\
\text { Gigaspora sp. } \\
\text { Glomus macrocarpum Tul. \& C. Tul. } \\
\text { Glomus sp. }\end{array}$ \\
\hline \multicolumn{5}{|c|}{ FF 2} \\
\hline $409 \mathrm{~B}$ & $\begin{array}{c}81,80 \text { B } \\
( \pm 11,43)\end{array}$ & $5 \mathrm{~A}$ & $\begin{array}{c}\text { Acaulosporaceae } \\
\text { Gigasporaceae } \\
\text { Glomeraceae }\end{array}$ & $\begin{array}{l}\text { Acaulospora foveata Trappe \& Janos } \\
\text { Acaulospora sp. } \\
\text { Gigaspora sp. } \\
\text { Glomus macrocarpum Tul. \& C. Tul. } \\
\text { Glomus sp. }\end{array}$ \\
\hline
\end{tabular}

${ }^{1}$ Médias seguidas de letras distintas na coluna são significativamente diferentes (teste $\mathrm{U}$ de Mann-Whitney, $\left.\mathrm{p}<0,05\right)$. Número total de esporos (NT); abundância de esporos em $50 \mathrm{~g}$ de solo (AB); riqueza de espécies (S).

a ausência de diferenças entre FF 1 e FF 2 quanto à maioria dos atributos químicos do solo (Tabela 1), já que estas variáveis podem influenciar a abundância de esporos e a riqueza de espécies de FMA, conforme constatado em dunas arenosas costeiras em três diferentes estágios de estabilização (Cordoba et al., 2001) e em um mesmo estágio de estabilização (Beena et al., 2000; Stürmer et al., 2013).

Os elevados teores de COT (Tabela 1) indicaram que o solo nas áreas estudadas é de constituição orgânica $\left(\mathrm{COT}>80 \mathrm{~g} \mathrm{~kg}^{-1}\right)$ (EMBRAPA, 2006). O importante acúmulo de matéria orgânica na superfície do solo e a decomposição lenta (Paula et al., 2009) foram responsáveis por isto. Nessa situação, os radicais carboxílicos, principalmente, e fenólicos sofrem hidrólise e acarretam em elevados teores de $\mathrm{H}^{+}$(Plieski et al., 2004). Como consequência, observou-se baixos valores de $\mathrm{pH}$ em ambos os fragmentos florestais (Tabela 1).

Em solos com pH ácido entre 4,5 e 4,8, a ocorrência de algumas espécies de FMA pode ser favorecida, como é o caso de espécies do gênero Glomus (Borba \& Amorim, 2007). O presente estudo corroborou essa afirmação, uma vez que esporos de Glomus macrocarpum e Glomus sp. foram os mais frequentemente encontrados nas florestas periodicamente inundáveis de Restinga na Marambaia ( $100 \%$ e $80 \%$ de todas as amostras analisadas, respectivamente). Contudo, este resultado divergiu do encontrado em dunas fixas na Praia da Joaquina, onde predominaram espécies de Glomus, apesar do pH igual a 5,9 e, portanto, consideravelmente menos ácido (Cordoba et al., 2001).

As espécies de Acaulospora (Acaulospora sp., Acaulospora foveata e Acaulospora scrobiculata) apresentaram a menor freqüência (50\%, 20\% e 10\%, respectivamente) entre as espécies dos demais gêneros de FMA encontrados, contudo foi o gênero com maior riqueza de espécies. Por outro lado, Gigaspora foi o gênero com menor riqueza, uma vez que apresentou apenas a espécie Gigaspora sp., e com freqüência intermediária (60\%) nas áreas do presente trabalho, em comparação com Glomus e Acaulospora.

Acaulospora e Glomus foram os gêneros com maior riqueza de espécies de FMA, nos fragmentos florestais em Marambaia (Tabela 2). Este resultado corroborou as informações levantadas em outros diferentes ecossistemas arenosos costeiros (Tabela 3), segundo os quais, de uma maneira geral, estes gêneros concentraram a maior riqueza de espécies, principalmente no caso de Glomus. As condições edáficas interferem na dominância de famílias e/ou gêneros de FMA, como no caso de dunas fixas, onde o teor de matéria orgânica e a disponibilidade de nutrientes foram maiores e houve predomínio da família Glomeraceae, representada pelo gênero Glomus, em detrimento de dunas incipientes e frontais na Praia da Joaquina, Estado de Santa Catarina (Cordoba et al., 2001). Por outro lado, em dunas frontais localizadas em diferentes sítios também em Santa Catarina, onde o teor de matéria orgânica foi baixo e o $\mathrm{pH}>6,0$, 
Tabela 3. Valores de abundância total média de esporos $\left(A B, n^{\circ} \mathrm{g}^{-1}\right.$ solo), riqueza total (S) e distribuição percentual de espécies de fungos micorrízicos arbusculares por gênero em diferentes ecossistemas de restinga.

Table 3. Values of total average abundance of spores $\left(A B, n^{\circ} \mathrm{g}^{-1}\right.$ soil), total richness $(S)$ and percentage distribution of AMF species by gender in different coastal ecosystems.

\begin{tabular}{|c|c|c|c|c|c|c|}
\hline \multirow{3}{*}{ Local } & \multirow{2}{*}{$\begin{array}{c}\text { AB } \\
\left(n^{0} \mathbf{g}^{-1}\right. \\
\text { solo }) \\
\end{array}$} & \multirow[b]{2}{*}{$\mathbf{S}$} & \multicolumn{3}{|c|}{ Gênero (\% da riqueza total) } & \multirow[b]{2}{*}{ Referência } \\
\hline & & & $\begin{array}{c}\text { Acaulos- } \\
\text { pora }\end{array}$ & Gigaspora & Glomus & \\
\hline & \multicolumn{6}{|c|}{ Florestas de restinga periodicamente inundáveis } \\
\hline \multirow{2}{*}{ Marambaia, RJ } & $5,13^{+}$ & $6^{+}$ & $50 \%^{+}$ & $17 \%^{+}$ & $33 \%^{+}$ & \multirow{2}{*}{ Presente estudo } \\
\hline & $1,64^{++}$ & $5^{++}$ & $40 \%^{++}$ & $20 \%{ }^{++}$ & $40^{++}$ & \\
\hline \multicolumn{7}{|c|}{ Florestas de restinga não-inundáveis } \\
\hline Ilha do Cardoso, SP & 0,50 & 35 & $34 \%$ & $3 \%$ & $34 \%$ & Trufem (1990b) \\
\hline \multirow{2}{*}{ Mataraca, PBi } & $0,26^{+++}$ & 10 & $10 \%$ & $20 \%$ & $40 \%$ & Souza et al. (2013) \\
\hline & - & 14 & $7 \%$ & $21 \%$ & $21 \%$ & Silva et al. (2012) \\
\hline \multicolumn{7}{|c|}{ Transição floresta-duna } \\
\hline Ilha do Cardoso, SP & 1,93 & 47 & $28 \%$ & $6 \%$ & $40 \%$ & Trufem (1995) \\
\hline \multicolumn{7}{|c|}{ Dunas arenosas costeiras } \\
\hline \multirow{2}{*}{ Ilha do Cardoso, SP } & - & 14 & $14 \%$ & $7 \%$ & $21 \%$ & Trufem et al. (1989) \\
\hline & 0,69 & 39 & $26 \%$ & $5 \%$ & $31 \%$ & Trufem (1990a) \\
\hline Goa, Îndia & 7,25 & 8 & $38 \%$ & $38 \%$ & $0 \%$ & Jaiswal \& Rodrigues (2001) \\
\hline \multirow{3}{*}{ Karnataka, Índia } & 0,75 & 16 & $0 \%$ & $19 \%$ & $56 \%$ & Kulkarni et al. (1997) \\
\hline & 0,57 & 41 & $17 \%$ & $10 \%$ & $44 \%$ & Beena et al. (2000) \\
\hline & 0,46 & 30 & $10 \%$ & $10 \%$ & $53 \%$ & Beena et al. (2001) \\
\hline \multirow{3}{*}{ Praia da Joaquina, SC } & $0,20^{*}$ & $6^{*}$ & $33 \%{ }^{*}$ & $17 \%^{*}$ & $0 \%^{*}$ & \multirow{3}{*}{ Cordoba et al. (2001) } \\
\hline & $1,70^{\star *}$ & $7^{* *}$ & $43 \%^{\star *}$ & $14 \%^{* *}$ & $14 \%^{* *}$ & \\
\hline & $2,15^{\star * *}$ & $9^{* * *}$ & $22 \% * * *$ & $11 \%^{* * *}$ & $56 \%^{* * *}$ & \\
\hline Praia do Cassino, RS & - & 10 & $10 \%$ & $20 \%$ & $20 \%$ & Cordazzo \& Stürmer (2007) \\
\hline $\begin{array}{l}\text { Itapiruba, Joaquina e } \\
\text { Praia Grande, SC }\end{array}$ & $0,30^{++++}$ & 25 & $20 \%$ & $8 \%$ & $20 \%$ & Stürmer et al. (2013) \\
\hline
\end{tabular}

${ }^{+} \mathrm{FF} 1,{ }^{++} \mathrm{FF} 2 ;{ }^{+++}$média das estações seca e chuvosa; ${ }^{++++}$média das três áreas; ${ }^{*}$ dunas incipientes; ${ }^{* *}$ dunas frontais; ${ }^{* * *}$ dunas fixas.

predominou a família Gigasporaceae, representada principalmente pelos gêneros Scutellospora e Racocetra, além de Gigaspora (Stürmer et al., 2013).

O número total de esporos extraídos do solo no FF 1 foi aproximadamente três vezes maior quando comparado ao FF 2. Isto se refletiu na abundância média de esporos de FMA significativamente maior no FF 1 (Tabela 2). Tal padrão pode ter ocorrido por dois principais motivos: os valores médios da disponibilidade de P e do teor de água no solo no FF 1 , que foram significativamente menores em relação ao observado em FF 2 (Tabela 1).

Em condições de maiores teores de P no solo, tende a ser menor a formação de micorriza e, portanto, é menor a presença de FMA no solo (Moreira \& Siqueira, 2006). Além disso, as condições de encharcamento a que os solos são periodicamente submetidos, com a consequente deficiência de oxigenação do solo, pode não só tornar pequena a população de FMA como pode até mesmo levar ao seu desaparecimento (Miranda \& Miranda, 1997), uma vez que os FMA são microrganismos aeróbicos (Slankis, 1974). Os resultados para o teor de água no solo já eram esperados, conforme dados anteriormente levantados na descrição das áreas estudadas (Paula et al., 2009).

A abundância de esporos de FMA apresentou correlação significativa para alguns atributos edáficos. Dentre estes, a correlação positiva foi verificada somente para o teor de água no solo superficial, em ambos os fragmentos florestais estudados na Restinga da Marambaia. Em contrapartida, a abundância de esporos de FMA correlacionou-se negativamente com a temperatura do solo, nas duas áreas; os valores de acidez potencial $\left(\mathrm{H}^{+}+\mathrm{Al}^{+3}\right)$, acidez ativa $\left(\mathrm{H}^{+}\right)$e $\mathrm{Ca}^{+2}$, em FF 1; e os valores de $\mathrm{Al}^{+3}, \mathrm{Mg}^{+2}$ e $\mathrm{P}$, em FF 2 (Tabela 4). Os atributos edáficos podem influenciar a formação de esporos de FMA de diferentes maneiras, entretanto nem sempre existe uma correlação positiva 
Tabela 4. Valores do coeficiente de correlação de Pearson entre a abundância de esporos (número de esporos $50 \mathrm{~g}^{-1}$ solo) de fungos micorrízicos arbusculares (FMA) e os atributos edáficos em dois fragmentos florestais (FF 1 e FF 2) de Restinga periodicamente inundável em Marambaia, RJ.

Table 4. Values of the Pearson correlation coefficient between spore abundance (number of spores $50 \mathrm{~g}^{-1}$ soil) of arbuscular mycorrhizal fungi (AMF) and soil attributes in two forest fragments (FF 1 and FF 2) of periodically flooded Restinga at Marambaia, RJ.

\begin{tabular}{|c|c|c|c|}
\hline \multirow{2}{*}{ Atributos edáficos } & & \multicolumn{2}{|c|}{ Áreas } \\
\hline & & FF 1 & FF 2 \\
\hline $\mathrm{H}+\mathrm{Al}$ & \multirow{8}{*}{ Abundância de esporos de FMA } & $-0,9934^{* *}$ & $-0,8965$ \\
\hline $\mathrm{H}^{+}$ & & $-0,9939 * *$ & $-0,8894$ \\
\hline $\mathrm{Al}$ & & $-0,6186$ & $-0,9843$ * \\
\hline $\mathrm{Ca}$ & & $-0,9959$ ** & $-0,8309$ \\
\hline $\mathrm{Mg}$ & & $-0,5409$ & $-0,9964^{* *}$ \\
\hline $\mathrm{P}$ & & $-0,9776$ & $-0,9997^{* *}$ \\
\hline Água & & $1,0000^{* *}$ & $1,0000^{* *}$ \\
\hline Temperatura & & $-1,0000^{* *}$ & $-1,0000^{* *}$ \\
\hline
\end{tabular}

*Significativo a $5 \%$ de probabilidade pelo teste F; ${ }^{*}$ Significativo a $1 \%$ de probabilidade pelo teste $\mathrm{F}$

entre a esporulação e a colonização radicular por FMA, embora um maior número de esporos no solo possa aumentar as chances de sua germinação e posterior formação da micorriza (Beena et al., 2000; Oliveira \& Oliveira, 2005).

Em algumas florestas tropicais, os maiores valores de abundância de esporos de FMA ocorreram em solos com temperaturas mais elevadas (Nandakwang et al., 2008; Sharma \& Jha, 2014). No presente trabalho, conforme anteriormente relatado, constatou-se o oposto, em função da correlação negativa entre a temperatura do solo e a abundância de esporos de FMA nas duas áreas, além do fato de que a maior abundância de esporos de FMA ocorreu em FF 1, onde a temperatura média do solo foi significativamente menor, em relação a FF 2 (Tabelas 1, 2 e 4). A divergência entre os resultados obtidos no presente trabalho e os observados naqueles anteriormente citados pode ter ocorrido em função das diferenças entre os ecossistemas florestais quanto aos demais atributos edáficos, tais como disponibilidade de $\mathrm{P}$ e conteúdo de água no solo, e à composição de espécies da comunidade de plantas.

A variação na riqueza de espécies de FMA nos ecossistemas depende da sua capacidade de adaptação às condições físicas e químicas vigentes (Theodoro et al., 2003), bem como da ocorrência e/ou predominância de hospedeiros distintos no ambiente, visto que existem variações de seletividade entre hospedeiro e fungo (Pouyu-Rojas et al., 2006). Portanto, a baixa diversidade da cobertura vegetal (Paula et al., 2009) e os atributos edáficos em FF 1 e FF 2 (Tabela 1), principalmente em se tratando da condição de anaerobiose, podem ser apontados como os responsáveis pela baixa riqueza de espécies e elevada abundância de esporos de FMA nestas áreas, em relação a florestas não-inundáveis, áreas de transição floresta-duna e dunas arenosas costeiras (Tabela 3). Da mesma forma, a ocorrência de Acaulospora scrobiculata apenas em FF 1 sugeriu que o elevado teor de água no solo em FF 2, que foi aproximadamente o dobro do observado na primeira área (Tabela 1), influenciou este resultado.

\section{CONCLUSÕES}

Os fragmentos florestais de Restinga periodicamente inundável em Marambaia, RJ, não diferiram entre si quanto à riqueza de espécies de fungos micorrízicos arbusculares, pois ambos apresentaram um total de seis espécies. O gênero Acaulospora, que apresentou maior riqueza de espécies, e o gênero Glomus, encontrado mais frequentemente, foram aqueles mais adaptados às condições dos ecossistemas estudados. A abundância de esporos foi maior no fragmento florestal com menor grau de saturação hídrica do solo, menor valor de $\mathrm{P}$ disponível, e de temperatura do solo, onde Acaulospora scrobiculata se restringiu.

\section{STATUS DA SUBMISSÃO}

Recebido: 30 dez., 2013

Aceito: 12 set., 2015 
AUTOR(ES) PARA CORRESPONDÊNCIA

\section{Marcos Gervasio Pereira}

Departamento de Solos, Universidade Federal Rural do Rio de Janeiro - UFRRJ, BR 465, Km 7, CEP 23897-970, Seropédica, RJ, Brasil e-mail: gervasio@ufrrj.br

\section{REFERÊNCIAS}

Allen MF, Hipps LE, Wooldridge G. Wind dispersal and subsequent establishment of VA mycorrhizal fungi across a successional arid landscape. Landscape Ecology 1989; 2(3): 165-171. http://dx.doi.org/10.1007/BF00126016.

Beena KR, Arun AB, Raviraja NS, Sridhar KR. Association of arbuscular mycorrhizal fungi with plants of coastal sand dunes of west coast of India. Tropical Ecology 2001; 42(2): 213-222.

Beena KR, Raviraja NS, Sridhar KR. Seasonal variations of arbuscular mycorrhizal fungal association with lpomoea pes-caprae of coastal sand dunes, Southern India. Journal of Environmental Biology 2000; 21(4): 341-347.

Borba MF, Amorim SMC. Fungos micorrízicos arbusculares em sempre-vivas: subsídio para cultivo e replantio em áreas degradadas. Revista de Biologia e Ciências da Terra 2007; 7(2): 20-27.

Brundrett MC. Mycorrhizal associations and other means of nutrition of vascular plants: understanding the global diversity of host plants by resolving conflicting information and developing reliable means of diagnosis. Plant and Soil 2009; 320(1-2): 37-77. http://dx.doi.org/10.1007/ s11104-008-9877-9.

Carneiro RFV, Martins MA, Freitas MSM, Detmann E, Vásquez HM. Bagaço de cana-de-açúcar como substrato para multiplicação de fungos micorrízicos arbusculares e sua influência sobre o estilosantes. Revista Caatinga 2008; 21(5): 189-196.

Cordazzo CV, Stürmer SL. Ocorrência de fungos micorrízicos arbusculares em Panicum racemosum (P. Beauv.) Spreng (Poaceae) em dunas costeiras do extremo sul do Brasil. Atlantica 2007; 29(1): 65-68.

Cordoba AS, Mendonça MM, Stürmer SL, Rygiewicz PT. Diversity of arbuscular mycorrhizal fungi along a sand dune stabilization gradient: A case study at Praia da Joaquina, Ilha de Santa Catarina, South Brazil. Mycoscience 2001; 42(4): 379-387. http://dx.doi.org/10.1007/BF02461221.

Dias PC, Pereira MSF, MegumiKasuya MC, Paiva HN, Oliveira LS, Xavier A. Micorriza arbuscular e rizóbios no enraizamento e nutrição de mudas de angico-vermelho. Revista Árvore 2012; 36(6): 1027-1037. http://dx.doi. org/10.1590/S0100-67622012000600004.
Egan C, Li DW, Klironomos J. Detection of arbuscular mycorrhizal fungal spores in the air across different biomes and ecoregions. Fungal Ecology 2014; 12: 26-31. http:// dx.doi.org/10.1016/j.funeco.2014.06.004.

Empresa Brasileira de Pesquisa Agropecuária - EMBRAPA. Centro Nacional de Pesquisas em Solos. Manual de métodos de análise de solos. Rio de Janeiro: EMBRAPA; 1997.212 p.

Empresa Brasileira de Pesquisa Agropecuária - EMBRAPA. Centro Nacional de Pesquisa de Solos. Sistema brasileiro de classificação de solos. 2. ed. Rio de Janeiro: EMBRAPA; 2006. $286 \mathrm{p}$.

Engel VL, Parrotta JA. Definindo a restauração ecológica: tendências e perspectivas mundiais. In: Kageyama PY, Oliveira RE, Moraes LFD, Engel VL, Gandara FB, organizadores. Restauração ecológica de ecossistemas naturais. Botucatu: FEPAF; 2003. p. 3-26.

Gerdemann JW, Nicolson TH. Spores of mycorrhizal endogone species extracted from soil by wet sieving and decanting. Transactions of the British Mycological Society 1963; 46(2): 235-244. http://dx.doi.org/10.1016/S00071536(63)80079-0.

Giri B, Kapoor R, Mukerji KG. Improved tolerance of Acacia nilotica to salt stress by arbuscular mycorrhiza, Glomus fasciculatum may be partly related to elevated $\mathrm{K} / \mathrm{Na}$ ratios in root and shoot tissues. Microbial Ecology 2007; 54(4): 753-760. http://dx.doi.org/10.1007/s00248007-9239-9. PMid:17372663.

Google Earth. Mapas [online]. 2014. [citado em 2014 dez. 22]. Disponível em: http://mapas.google.com

Jaiswal V, Rodrigues BF. Occurrence and distribution of arbuscular mycorrhizal fungi in coastal sand dune vegetation of Goa. Current Science 2001; 80(7): 826-827.

Jenkins WR. A rapid centrifugal-flotation technique for separating nematodes from soil. Plant Disease Reporter 1964; 48(9): 692.

Köppen W. Climatologia: con un estudio de los climas de La tierra. México: Fondo de Cultura Econômica; 1948. 479 p.

Kulkarni SS, Raviraja NS, Sridhar KR. Arbuscular mycorrhizal fungi on tropical sand dunes of West Coast of India. Journal of Coastal Research 1997; 13(3): 931-936.

Mattos CLV. Caracterização climática da Restinga da Marambaia. In: Menezes LFT, Peixoto AL, Araújo DSD, editores. História natural da Marambaia. Seropédica: EDUR; 2005. p. 55-66.

Mello AH, Santos ER, Nunes JS, Knoechelmann CM, Bezerra J, Michelotti F. Produção de mudas de espécies arbóreas inoculadas com fungos micorrízicos para atuar na reabilitação de áreas impactadas pela extração de argila. Agroecossistemas 2011; 3(1): 78-82.

Menezes LFT, Araújo DSD. Formações vegetais da Restinga da Marambaia. In: Menezes LFT, Peixoto AL, Araújo DSD, editores. História natural da Marambaia. Seropédica: EDUR; 2005. p. 67-120. 
Miranda JCC, Miranda LN. Micorriza arbuscular. In: Vargas MAT, Hungria M, editores. Biologia dos solos dos cerrados. Planaltina: EMBRAPA; CPAC; 1997. p. 69-132.

Moreira FMS, Siqueira JO. Microbiologia e bioquímica do solo. Lavras: UFLA; 2006. 729 p.

Nandakwang P, Elliott S, Youpensuk S, Lumyong S. Effects of arbuscular mycorrhizal inoculation and fertilizer on production of Castanopsis acuminatissima saplings for forest restoration in Northern Thailand. Research Journal of Microbiology 2008; 3: 225-236. http://dx.doi.org/10.3923/ jm.2008.225.236

Oliveira AN, Oliveira LA. Seasonal dynamics of arbuscular mycorrhizal fungi in plants of Theobroma grandiflorum Schum and Paullinia cupana Mart. of an agroforestry system in Central Amazonia, Amazonas state, Brazil. Brazilian Journal of Microbiology 2005; 36(3): 262-270. http://dx.doi.org/10.1590/S1517-83822005000300011.

Paula RR, Pereira MG, Menezes LFT. Aporte de nutrientes e decomposição da serapilheira em três fragmentos florestais periodicamente inundados na Ilha da Marambaia, RJ. Ciência Florestal 2009; 19(2): 139-148. http://dx.doi. org/10.5902/19805098405.

Plieski GLA, Ebeling AG, Anjos LHC, Pereira MG, Valladares GS. Avaliação de métodos analíticos para determinar acidez em solos com alto teor de matéria orgânica. Revista Universidade Rural: Série Ciências da Vida 2004; 24(2): 15-21.

Pouyu-Rojas E, Siqueira JO, Santos JGD. Compatibilidade simbiótica de fungos micorrízicos arbusculares com espécies arbóreas tropicais. Revista Brasileira de Ciencia do Solo 2006; 30(3): 413-424. http://dx.doi.org/10.1590/ S0100-06832006000300003.

Qiangsheng W, Renxue X, Zhengjia H. Effect of arbuscular mycorrhiza on the drought tolerance of Poncirus trifoliata seedlings. Frontiers of Forestry in China 2006; 1(1): 100104. http://dx.doi.org/10.1007/s11461-005-0007-z.

Rodríguez-Echeverría S, Freitas H. Diversity of AMF associated with Ammophila arenaria ssp. arundinacea in Portuguese sand dunes. Mycorrhiza 2006; 16(8): 543-552. http://dx.doi.org/10.1007/s00572-006-0070-9. PMid:17043895.

Roncarati H, Menezes LFT. Marambaia, Rio de Janeiro: origem e evolução. In: Menezes LFT, Peixoto AL, Araujo DSD, organizadores. História Natural da Marambaia. Seropédica: Editora da Universidade Federal Rural do Rio de Janeiro; 2005. p. 15-38.

Santos DR, Costa MCS, Miranda JRP, Santos RV. Micorriza e rizóbio no crescimento e nutrição em $\mathrm{N}$ e P em mudas de angico-vermelho. Revista Caatinga 2008; 21(1): 76-82.

Schiavo JA, Martins MA, Rodrigues LA. Avaliação nutricional de mudas de Acacia mangium, Sesbania virgata e Eucalyptus camaldulensis inoculadas com fungos micorrízicos, em casa-de-vegetação e em cava de extração de argila. Acta Scientiarum. Agronomy 2009; 31(4): 701 707. http://dx.doi.org/10.4025/actasciagron.v31i4.1364.

Sharma B, Jha DK. Arbuscular mycorrhizal fungal association in some commonly occurring economically important plants of Kaziranga National Park, Assam, India. Indian Phytopathology 2014; 67(3): 243-251.

Silva DKA, Pereira CMR, Souza RG, Silva GA, Oehl F, Maia LC. Diversity of arbuscular mycorrhizal fungi in restinga and dunes areas in Brazilian Northeast. Biodiversity and Conservation 2012; 21(9): 2361-2373. http://dx.doi. org/10.1007/s10531-012-0329-8.

Slankis V. Soil factors influencing formation of mycorrhizae. Annual Review of Phytopathology 1974; 12(1): 437-457. http://dx.doi.org/10.1146/annurev.py.12.090174.002253.

Souza RC, Pereira MG, Giácomo RG, Silva EMR, Menezes LFT. Produção de mudas micorrizadas de Schinus terebinthifolius Raddi. em diferentes substratos. Floresta 2009; 39(1): 197-206. http://dx.doi.org/10.5380/ rf.v39i1.13739.

Souza RG, Silva DKA, Mello CMA, Goto BT, Silva FSB, Sampaio EVSB et al. Arbuscular mycorrhizal fungi in revegetated mined dunes. Land Degradation \& Development 2013; 24(2): 147-155. http://dx.doi.org/10.1002/ldr.1113.

Stürmer SL, Stürmer R, Pasqualini D. Taxonomic diversity and community structure of arbuscular mycorrhizal fungi (Phylum Glomeromycota) in three maritime sand dunes in Santa Catarina state, south Brazil. Fungal Ecology 2013; 6(1): 27-36. http://dx.doi.org/10.1016/j.funeco.2012.10.001.

Tavares RC, Mendes Filho PF, Lacerda CF, Silva J. Colonização micorrízica e nodulação radicular em mudas de sabiá (Mimosa caesalpiniaefolia Benth.) sob diferentes níveis de salinidade. Revista Ciência Agronômica 2012; 43(3): 409416. http://dx.doi.org/10.1590/S1806-66902012000300001.

Theodoro VCA, Alvarenga MIN, Guimarães RJ, Mourão $\mathrm{M}$ Jr. Carbono da biomassa microbiana e micorriza em solo sob mata nativa e agroecossistemas cafeeiros. Acta Scientiarum. Agronomy 2003; 25(1): 147-153. http://dx.doi. org/10.4025/actasciagron.v25i1.2468.

Trufem SFB, Otomo HS, Malatinsky SMM. Fungos micorrízicos vesículo-arbusculares em rizosferas de plantas em dunas do Parque Estadual da Ilha do Cardoso, São Paulo, Brasil. (1) Taxonomia. Acta Botanica Brasílica 1989; 3(2): 141-152. http://dx.doi.org/10.1590/S010233061989000300014 .

Trufem SFB. Aspectos ecológicos de fungos micorrízicos arbusculares na rizosfera de plantas de restinga da Ilha do Cardoso, SP, Brasil. Revista Brasileira de Botanica. Brazilian Journal of Botany 1995; 18(1): 51-60.

Trufem SFB. Aspectos ecológicos de fungos micorrízicos vesículo-arbusculares em ecossistema de dunas no Parque Estadual da Ilha do Cardoso, SP, Brasil. In: Anais do II Simpósio de Ecossistemas da Costa Sul e Sudeste Brasileira: 
estrutura, função e manejo; 1990a; São Paulo. São Paulo: ACIESP; 1990a. vol. 3. p. 478-487.

Trufem SFB. Aspectos ecológicos de fungos micorrízicos vesículo-arbusculares da mata tropical úmida da Ilha do Cardoso, SP, Brasil. Acta Botanica Brasílica 1990b; 4(2): 3145. http://dx.doi.org/10.1590/S0102-33061990000200003.

Wubet T, Kottke I, Teketay D, Oberwinkler F. Mycorrhizal status of indigenous trees in dry Afromontane forests of
Ethiopia. Forest Ecology and Management 2003; 179(1-3): 387-399. http://dx.doi.org/10.1016/S0378-1127(02)00546-7.

Yeomans JC, Bremner JM. A rapid and precise method for routine determination of organic carbon in soil. Communications in Soil Science and Plant Analysis 1988; 19(13): 1467-1476. http://dx.doi.org/10.1080/00103628809368027.

Zar JH. Biostatistical analysis. 3. ed. New Jersey: Prentice Hall; 1984. 662 p. 